\title{
Existence of weak solutions to SPDEs with fractional Laplacian and non-Lipschitz coefficients
}

\author{
Shohei Nakajima ${ }^{1}$ (D)
}

Received: 24 July 2019 / Revised: 13 February 2021 / Accepted: 24 May 2021 / Published online: 9 June 2021

(C) The Author(s) 2021

\section{Abstract}

We prove existence of solutions and its properties for a one-dimensional stochastic partial differential equations with fractional Laplacian and non-Lipschitz coefficients. The method of proof is eatablished by Kolmogorov's continuity theorem and tightness arguments.

Keywords Fractional Laplacian · Non-Lipschitz coefficients · Polynomial decay

\section{Introduction}

Stochastic partial differential equations with fractional Laplacian appear in many fields such as physics, fractal medium, image analysis, risk management and other fields (see Debbi [4], Mueller [12] and Xie [15]). In this paper, we discuss the existence and uniqueness of solutions to the initial value problem for the following stochastic partial differential equation which is denoted by SPDE:

$$
\left\{\begin{aligned}
\frac{\partial X}{\partial t}(t, x) & =-(-\Delta)^{\alpha / 2} X(t, x)+|X(t, x)|^{\gamma} \dot{W}(t, x), \quad t \geq 0, x \in \mathbb{R}, \\
X(0, x) & =f(x),
\end{aligned}\right.
$$

where $-(-\Delta)^{\alpha / 2}$ is the fractional Laplacian with order $1<\alpha<2, f$ is an initial value, $\dot{W}(t, x)$ is a space-time white noise on $[0, \infty) \times \mathbb{R}$, and $\gamma$ is a parameter satisfying $1 / 2<\gamma<1$.

Shohei Nakajima

st08m26@akane.waseda.jp

1 Department of Applied Mathmatics, Waseda University, Shinjuku, Japan 
We will explain some known results related to (1.1). Consider the case where $\alpha=2$ and coefficients of noise are Lipschitz continuous, that is,

$$
\left\{\begin{aligned}
\frac{\partial X}{\partial t}(t, x) & =\Delta X(t, x)+a(X(t, x)) \dot{W}(t, x), \quad t \geq 0, x \in \mathbb{R} \\
X(0, x) & =f(x)
\end{aligned}\right.
$$

where $a: \mathbb{R} \rightarrow \mathbb{R}$ is Lipschitz continuous. Equation (1.2) was studied by Funaki [7] and Walsh [18]. They showed the unique existence of a solution to (1.2) and spatial regularity of the solution. Later Mueller-Perkins [11] and Shiga [17] studied the SPDE (1.2) without assuming the Lipschitz continuty of coefficients $a$. They imposed the following growth condition on $a(u)$ :

$$
|a(u)| \leq C\left(|u|+|u|^{\theta}\right), \quad u \in \mathbb{R}
$$

for some $0<\theta<1$. They showed the existence of probability space $(\bar{\Omega}, \overline{\mathcal{F}}, \bar{P})$ on which there is a space-time white noise $\dot{\bar{W}}(t, x)$ such that $(1.2)$ with $\dot{W}(t, x)$ replaced by $\dot{\bar{W}}(t, x)$ has a mild solution $X(t, x)$. In addition, they proved that, if $f$ is positive, then any solution $X(t, x)$ to (1.2) is positive for all $t \geq 0$ and $x \in \mathbb{R}$. Moreover, it was shown that, if $f(x)$ is sufficiently rapidly decreasing as $x \rightarrow \infty$, then any solution $X(t, x)$ to (1.2) is also sufficiently rapidly decreasing. Mytnik [13] proved the weak uniqueness of solutions to the following equation which is a special one of (1.2):

$$
\frac{\partial X}{\partial t}(t, x)=\Delta X(t, x)+|X(t, x)|^{\gamma} \dot{W}(t, x), \quad t \geq 0, x \in \mathbb{R},
$$

with $1 / 2<\gamma<1$. The idea of his proof is based on a duality argument developed by Ethier-Kurtz [5]. Mytnik [14] studied the dual process $Y$ described by the following SPDE:

$$
\frac{\partial Y}{\partial t}(t, x)=\Delta Y(t, x)+|Y(t, x)|^{1 / \gamma} \dot{L}(t, x), \quad t \geq 0, x \in \mathbb{R},
$$

where $\dot{L}$ is a stable noise on $\mathbb{R} \times \mathbb{R}_{+}$with nonnegative jumps. He coordinated a probability space $(\tilde{\Omega}, \tilde{\mathcal{F}}, \tilde{P})$ on which there exists a stable noise $\dot{L}$ and random field $Y(t, x)$, where $Y(t, x)$ is a mild solution of (1.5).

Recently SPDEs with fractional Laplacian have been discussed by several authors (see; e.g. Chen [1], Debbi [4], Niu-Xie [15]). Consider (1.2) with $\Delta$ replaced by $-(-\Delta)^{\alpha / 2}$ for $1<\alpha<2$ and Lipschitz continuous coefficients of noise, that is,

$$
\left\{\begin{aligned}
\frac{\partial X}{\partial t}(t, x) & =-(-\Delta)^{\alpha / 2} X(t, x)+a(X(t, x)) \dot{W}(t, x), \quad t \geq 0, x \in \mathbb{R} \\
X(0, x) & =f(x)
\end{aligned}\right.
$$

where $a: \mathbb{R} \rightarrow \mathbb{R}$ is Lipschitz continuous. Chen [1], Debbi [4] and Niu-Xie [15] have shown the unique existence of mild solution to (1.6) and its regularity. 
However, it is still an open problem to show the existence and uniqueness of solution to (1.6) without assuming the Lipschitz continuty of $a(u)$. The purpose of this paper is to establish the existence and weak uniqueness of a mild solution to (1.1) which is a special case of (1.6) where $a(u)=|u|^{\gamma}$ with $\frac{1}{2}<\gamma<1$. We intend to use similar aguments to Mueller-Perkins [11], Shiga [17], and Mytnik [13]. The large difference between the fractional Laplacian and the usual Laplacian can be found in the decay properties of fundamental solutions as $x \rightarrow \infty$. Since the fundamental solution of the usual Laplacian has an exponential decay property, the solution $X(t, x)$ to (1.2) has the exponential decay property if the condition (1.3) holds. Similarly the fundamental solution of fractional Laplacian decays polynomially, we can show that the solution to (1.1) has the polinomial decay property if the condition (1.3) holds. To prove the uniqueness of solution to (1.4) Mytnik [13] used the exponential decay property. It is enough to use the polynomial decay property in order to prove the uniqueness of solution to (1.1).

This paper is organized as follows: in Sect. 2 we prepare some tools and lemma to prove our main results. In Sect. 3, we show the existence and uniqueness of solution by applying the Banach fixed point theorem with Lipschitz continuous coefficients $a(X)$. In addition, we follow the argument of Mueller-Perkins [11] and Shiga [17] to prove positivity and polynomial decay properties of solutions to (1.6). From Sect. 4 we begin with consideration (1.1). We prove the existence of solution to (1.1) by tightness arguments. In fact, we can prove a uniqueness of solution in the distributional sense by applying to duality method. Since the proof is almost same as the paper [5], we omit the detail.

\section{Preliminaries}

\subsection{Fractional differential operator}

For $1<\alpha \leq 2$, let $-(-\Delta)^{\alpha / 2}$ be a fractional differential operator defined by the Fourier transform $\mathcal{F}$ :

$$
\mathcal{F}\left((-\Delta)^{\alpha / 2} X\right)(\xi)=|\xi|^{\alpha} \mathcal{F}(X)(\xi), \quad X \in D\left((-\Delta)^{\alpha / 2}\right), \quad \xi \in \mathbb{R},
$$

where

$$
\mathcal{F}(X)(\xi)=\int_{\mathbb{R}} X(x) e^{-i x \xi} d x
$$

Let $G(t, x)$ be a fundamental solution to the Cauchy problem

$$
\left\{\begin{aligned}
\frac{\partial G}{\partial t}(t, x) & =-(-\Delta)^{\alpha / 2} G(t, x), \\
G(0, x) & =\delta_{0}(x)
\end{aligned}\right.
$$


where $\delta_{0}$ denotes the Dirac measure. Then $G(t, x)$ can be expressed using the Fourier transform:

$$
G(t, x)=\int_{\mathbb{R}} \exp \left\{-i \xi x-t|\xi|^{\alpha}\right\} d \xi
$$

and has the following properties (cf. Debbi-Dozzi [4] and Kotelenez [9]):

Lemma 2.1 Let $G(t, x)$ be the fundamental solution of (2.1) and define

$$
\lambda(x)=\left(1+|x|^{2}\right)^{\frac{1}{2}}, \quad x \in \mathbb{R} .
$$

Then there exists a constant $C_{\alpha}>0$ such that for all $0 \leq s \leq t \leq T, x \in \mathbb{R}$, and $0<\rho \leq(\alpha+1) / 2$, the following properties hold:

$$
\begin{aligned}
& \text { (i) } \frac{\partial^{n} G}{\partial x^{n}}(t, x)=\left.t^{-\frac{n+1}{\alpha}} \frac{\partial^{n} G(1, \xi)}{\partial \xi^{n}}\right|_{\xi=t^{-\frac{1}{\alpha}} x} . \\
& \text { (ii) } \int_{\mathbb{R}} G(t, x) \lambda^{\rho}(x) d x<\infty \\
& \text { (iii) }|G(1, x)| \leq C_{\alpha}\left(1+|x|^{1+\alpha}\right)^{-1} \\
& \text { (iv) }\left|\frac{\partial^{n} G}{\partial x^{n}}(1, x)\right| \leq C_{\alpha} \frac{1+|x|^{\alpha+n-1}}{\left(1+|x|^{\alpha+n}\right)^{2}}
\end{aligned}
$$

Hereafter, we sometimes write $G(t, x-y)=G(t, x, y)$. Note that there exists a constant $C_{1}, C_{2}>0$ such that $\lambda^{-\rho}(y) \leq C_{1} \lambda^{\rho}(x-y) \lambda^{-\rho}(x)$ and from Lemma 2.1 (ii) we obtain the estimate

$$
\int_{\mathbb{R}} G(t, x, y) \lambda^{-\rho}(y) d y \leq C_{2} \lambda^{-\rho}(x) .
$$

\subsection{Definition of the solution}

Let $\left(\Omega, \mathcal{F}, \mathcal{F}_{t}, P\right)$ be a complete probability space with filtration and $\dot{W}(t, x)$ be an $\left\{\mathcal{F}_{t}\right\}$-space-time Gaussian white noise with covariance given by

$$
E\left[\dot{W}(t, x) \dot{W}\left(t^{\prime}, x^{\prime}\right)\right]=\delta\left(t-t^{\prime}\right) \delta\left(x-x^{\prime}\right)
$$

for $t, t^{\prime} \geq 0$ and $x, x^{\prime} \in \mathbb{R}$. For an $\left\{\mathcal{F}_{t}\right\}$-predictable functional $\phi(t, x, \omega):[0, \infty) \times$ $\mathbb{R} \times \Omega \rightarrow \mathbb{R}$ satisfying

$$
E\left[\int_{0}^{t} \int_{\mathbb{R}} \phi^{2}(s, x, \omega) d x d s\right]<\infty \text { for all } t>0
$$


we can define stochastic integral (cf. Walsh [18])

$$
\int_{0}^{t} \int_{\mathbb{R}} \phi(s, x, \omega) W(d x, d s)
$$

with quadratic variational process

$$
\int_{0}^{t} \int_{\mathbb{R}} \phi^{2}(s, x, \omega) d x d s .
$$

The Eq. (1.1) makes sense if we integrate the equation in time and space and use the initial condition.

Definition 2.1 An $\left(\mathcal{F}_{t}\right)$-adapted random field $\{X(t, x), t \geq 0, x \in \mathbb{R}\}$ is said to be a solution in the sense of generalized functions of (1.1) if for any $\phi \in C_{0}^{\infty}(\mathbb{R})$, the following equality holds:

$$
\begin{aligned}
\int_{\mathbb{R}} X(t, x) \phi(x) d x= & \int_{\mathbb{R}} f(x) \phi(x) d x-\int_{0}^{t} \int_{\mathbb{R}} X(s, x)(-\Delta)^{\alpha / 2} \phi(x) d x d s \\
& +\int_{0}^{t} \int_{\mathbb{R}}|X(s, x)|^{\gamma} \phi(x) W(d x, d s) \text { a.s. }
\end{aligned}
$$

Using the Green function, we can describe a solution of (1.1) in a mild form.

Definition 2.2 An $\left(\mathcal{F}_{t}\right)$-adapted random field $\{X(t, x), t \geq 0, x \in \mathbb{R}\}$ is said to be a mild solution of (1.1) with initial function $f$ if the following stochastic integral equation holds:

$$
X(t, x)=\int_{\mathbb{R}} G(t, x, y) f(y) d y+\int_{0}^{t} \int_{\mathbb{R}} G(t-s, x, y)|X(s, y)|^{\gamma} W(d y, d s) \text { a.s. }
$$

where $G(t, x, y)$ denotes the Green function of (2.1).

We introduce a martingale problem induced by (1.1).

Definition 2.3 Let $S$ be a Banach space. A solution to martingale problem for (1.1) we mean a measurable process $X$ with values in $S$ defined on some probability space $\left(\Omega, \mathcal{F}, P,\left\{\mathcal{F}_{t}\right\}\right)$ with a filtration satisfyng for all $\phi \in \mathcal{D}(-\Delta)^{\alpha / 2}$

$$
\left\langle X_{t}, \phi\right\rangle-\left\langle X_{0}, \phi\right\rangle+\int_{0}^{t}\left\langle X_{s},(-\Delta)^{\alpha / 2} \phi\right\rangle d s
$$

is an $\mathcal{F}_{t}^{X}$ square integrable martingale with the quadratic variation given by

$$
\int_{0}^{t} \int_{\mathbb{R}}|X(s, y)|^{2 \gamma} \phi(y)^{2} d y d s
$$


Definition 2.4 Let $S$ be a Banach space and $X_{1}$ and $X_{2}$ be $S$-valued mild solutions to the SPDE (1.1) with the same initial value. We say that the SPDE (1.1) has pathwise uniqueness if

$$
P\left(\left|X_{1}(t, \cdot)-X_{2}(t, \cdot)\right|_{S}=0, \quad \forall t \geq 0\right)=1 .
$$

holds.

To this end, it is required that all the terms in (2.4) and (2.3) are well defined. Here, a relationship between a solution in the sense of generalized functions and a mild solution is well known (cf. [18]).

Proposition 2.1 A solution in the sense of generalized functions of (1.1) is equivalent to the mild solution.

A solution to the martigale problem and a mild solution in weak sense are equivalent (cf. [7]).

Proposition 2.2 The following (1) and (2) are equivalent.

(1) $X(t, x)$ is a solution to the martigale problem for (1.1).

(2) There exists an $\left\{\mathcal{F}_{t}\right\}$-space-time Gaussian white noise $\dot{W}(t, x)$ and stochastic process $X(t, x)$ such that $X(t, x)$ is a mild solution of the SPDE (1.1) on a suitable probability space with filtration $\left(\Omega, \mathcal{F}, P,\left\{\mathcal{F}_{t}\right\}\right)$.

\section{Some properties of solutions in Lipschitz case}

\subsection{Existence and uniqueness of mild solutions}

In order to prove the existence and uniqueness to SPDE (1.1), we first consider the case where coefficients are Lipschitz continuous:

$$
\frac{\partial X_{t}}{\partial t}=-(-\Delta)^{\alpha / 2} X_{t}+a\left(X_{t}\right) \dot{W}(t, x)
$$

where $a: \mathbb{R} \rightarrow \mathbb{R}$ is Lipschitz continuous. Using the Green function, we can write the solution of Eq. (3.1) in a mild form:

$$
X(t, x)=\int_{\mathbb{R}} G(t, x, y) f(y) d y+\int_{0}^{t} \int_{\mathbb{R}} G(t-s, x, y) a(X(s, y)) W(d y, d s)(3.2)
$$

For any $0<\rho<(\alpha+1) / 2$, define a weighted $L^{2}$-norm defined by

$$
\|X\|_{L_{\rho}^{2}}=\left(\int_{\mathbb{R}}|X(x)|^{2} \lambda^{-\rho}(x) d x\right)^{1 / 2}
$$


Theorem 3.1 Assume that $f \in L_{\rho}^{2}(\mathbb{R})$ and $a: \mathbb{R} \rightarrow \mathbb{R}$ is a Lipschitzfunction satisfying linear growth condition, that is, there exists $C>0$ such that

$$
|a(u)| \leq C(1+|u|), \quad \text { for all } u \in \mathbb{R} .
$$

Then (3.2) admits the pathwise unique mild solution $X(t, x)_{(t, x) \in[0, T] \times \mathbb{R}}$ such that for each $T>0$,

$$
\sup _{0 \leq t \leq T} E\left[\|X(t, \cdot)\|_{L_{\rho}^{2}}^{2}\right]<\infty
$$

For every $0<\rho<(\alpha+1) / 2$, define a function space

$$
C_{\rho}(\mathbb{R}):=\left\{g \in C(\mathbb{R}): \sup _{x \in \mathbb{R}} \lambda^{\rho}(x) g(x)<\infty\right\} .
$$

The regularity of solution to (3.1) is well known (cf. [4], [15]).

Theorem 3.2 Under the conditions of Theorem 3.1 with the initial condition $f \in$ $C_{\rho}(\mathbb{R})$, the solution $X(t, x)$ of $(3.1)$ has the continuous modification on $[0, \infty) \times \mathbb{R}$. In addition, the solution $X(t, x)$ to (3.1) has the $\beta$-Hölder continuous modification for $t \in[0, \infty)$ and $\eta$-Hölder continuous modification for $x \in \mathbb{R}$ with $\beta \in\left(0, \frac{\alpha-1}{2 \alpha}\right)$ and $\eta \in\left(0, \frac{\alpha-1}{2}\right)$.

\subsection{Positivity of solution}

We will follow Shiga's arguments [17] to prove the positivity of the solution to (3.1). At first, we need to prepare a boundness of the solution to (3.1).

Lemma 3.1 Assume that for every $T>0$ there exists $C_{T}>0$ and such that

$$
|a(u)| \leq C_{T}\left(|u|+|u|^{1 / 2}\right), \quad u \in \mathbb{R} .
$$

Then for $p \geq 1$ can be written by $p=2^{m}, m \in \mathbb{N} \cup\{0\}$ and $0<\rho<(\alpha+1) / 2$, the mild solution $X(t, x)$ to (3.2) with initial condition $f \in C_{\rho}(\mathbb{R})$ satisfies

$$
\sup _{0 \leq t \leq T} \int_{\mathbb{R}} E\left[|X(t, x)|^{p}\right] \lambda^{\rho}(x) d x<\infty .
$$

Proof Assume that $p=1$. Then for every $0<\rho<(\alpha+1) / 2$ and $t \geq 0$, we have

$$
\begin{aligned}
\int_{\mathbb{R}} E\left[|X(t, x)| \lambda^{\rho}(x) d x\right] & =\int_{\mathbb{R}}\left|\int_{\mathbb{R}} G(t, x, y) f(y) \lambda^{\rho}(x) d y\right| d x \\
& \leq C \int_{\mathbb{R}}|f(y)| \lambda^{\rho}(y) d y<\infty .
\end{aligned}
$$


We will apply an induction argument. For $p \geq 2$, using the Burkhorder's inequality, the Hölder's inequality shows that

$$
\begin{aligned}
& E\left[|X(t, x)|^{p}\right] \\
& \leq C_{p}\left\{\left|\int_{\mathbb{R}} G(t, x, y) f(y) d y\right|^{p}+E\left|\int_{0}^{t} \int_{\mathbb{R}} G(t-s, x, y)^{2} a(X(s, y))^{2} d y d s\right|^{p / 2}\right\} \\
& \leq C_{p, T}\left\{\left|\int_{\mathbb{R}} G(t, x, y) f(y) d y\right|^{p}+E \int_{0}^{t} \int_{\mathbb{R}} G(t-s, x, y)^{2}|a(X(s, y))|^{p} d y d s\right\} . \\
& \leq C_{p, T}\left\{\left|\int_{\mathbb{R}} G(t, x, y) f(y) d y\right|^{p}\right. \\
& \left.\quad+E \int_{0}^{t} \int_{\mathbb{R}} G(t-s, x, y)^{2}\left[|X(s, y)|^{p}+|X(s, y)|^{p / 2}\right] d y d s\right\} .
\end{aligned}
$$

Multiply both sides by $\lambda^{\rho}(x)$ and integrate with variable $x$, we obtain that

$$
\begin{aligned}
& \int_{\mathbb{R}} E\left[|X(t, x)|^{p}\right] \lambda^{\rho}(x) d x \\
& \leq C_{p, T}\left\{1+E \int_{0}^{t} \int_{\mathbb{R}}\left[|X(s, y)|^{p}+|X(s, y)|^{p / 2}\right] \int_{\mathbb{R}} G(t-s, x, y)^{2} \lambda^{\rho}(x) d x d y d s\right\} \\
& \leq C_{p, T}\left\{1+E \int_{0}^{t} \int_{\mathbb{R}}\left[|X(s, y)|^{p}+|X(s, y)|^{p / 2}\right](t-s)^{-1 / \alpha} \lambda^{\rho}(y) d y d s\right\} \\
& \leq C_{p, T}\left\{1+\sup _{0 \leq s \leq t} \int_{\mathbb{R}} E\left[|X(s, y)|^{p}+|X(s, y)|^{p / 2}\right] \lambda^{\rho}(y) d y \int_{0}^{t}(t-s)^{-1 / \alpha} d s\right\} .
\end{aligned}
$$

Setting $p=2$, (3.6) and Grownwall's lemma imply that

$$
\sup _{0 \leq t \leq T} \int_{\mathbb{R}} E\left[|X(t, x)|^{2}\right] \lambda^{\rho}(x) d x<\infty
$$

From an induction argument, we complete the proof for $p=2^{m}$ with $m \in \mathbb{N} \cup\{0\}$.

Remark 3.1 We can get in a similarly way to the proof of Lemma 3.1 and Hölder's inequality together,

$$
\sup _{0 \leq t \leq T} \sup _{x \in \mathbb{R}} E\left[|X(t, x)|^{p} \lambda(x)^{\rho}\right]<\infty \text { for all } p \geq 2
$$

For any $0<\rho<(\alpha+1) / 2$ define the function space $C_{\rho}^{+}(\mathbb{R})$ by

$$
C_{\rho}^{+}(\mathbb{R}):=\left\{g \in C(\mathbb{R}): g \geq 0, \sup _{x \in \mathbb{R}} \lambda^{\rho}(x) g(x)<\infty\right\} .
$$


Theorem 3.3 Let $X(t, x)$ be the mild solution of (3.2) with the initial function $f \in$ $C_{\rho}^{+}(\mathbb{R})$. Assume that $a(u)$ satisfies the condition (3.4). In particular, $a(0)=0$. Then, we have

$$
P(X(t, x) \geq 0 ; t \geq 0, x \in \mathbb{R})=1 .
$$

Proof For every $\varepsilon>0$, choose non-negative, symmetric and smooth function $\rho_{\varepsilon}(x)$ such that

$$
\rho_{\varepsilon}(x)=0 \text { for }|x| \geq \varepsilon \text { and } \int_{\mathbb{R}} \rho_{\varepsilon}^{2}(x) d x=1 .
$$

Define

$$
W_{x}^{\varepsilon}(t)=\int_{0}^{t} \int_{\mathbb{R}} \rho_{\varepsilon}(x-y) W(d y, d s) .
$$

Notice that, for every $x \in \mathbb{R}, W_{x}^{\varepsilon}(t)$ is a one-dimensional Brownian motion. Set $\Delta_{\varepsilon}=(G(\varepsilon)-I) / \varepsilon$ for $\varepsilon>0$ where $G(\varepsilon) f(x)=(G(\varepsilon, \cdot) * f)(x)$. We consider the following equation

$$
X_{\varepsilon}(t, x)=f(x)+\int_{0}^{t}\left(\Delta_{\varepsilon} X_{\varepsilon}(s, x)\right) d s+\int_{0}^{t} a\left(X_{\varepsilon}(s, x)\right) d W_{x}^{\varepsilon}(s) .
$$

Since $a(u)$ is Lipschitz continuous and $\Delta_{\varepsilon}$ is a bounded operator on $L^{2}(\mathbb{R})$, the above equation has the unique strong solution and continuous version(cf. [2]). From now on, we claim that

$$
P\left(X_{\varepsilon}(t, \cdot) \geq 0, \quad \forall t \geq 0\right)=1 .
$$

Let $a_{n}=-2\left(n^{2}+n+2\right)^{-1}$ be a non-increasing sequence. Immediately, we obtain that $a_{n} \rightarrow 0$ as $n \rightarrow \infty$ and $\int_{a_{n-1}}^{a_{n}} x^{-2} d x=n$. Let $\psi_{n}(x)$ be a nonnegative continuous function such that

$$
\operatorname{supp}\left(\psi_{n}\right)=\left(a_{n-1}, a_{n}\right), \quad 0 \leq \psi_{n}(x) \leq \frac{2}{n x^{2}} \text { and } \int_{a_{n-1}}^{a_{n}} \psi_{n}(x) d x=1 .
$$

Define

$$
\Phi_{n}(x)=\int_{0}^{x} \int_{0}^{y} \psi_{n}(z) d z d y
$$

Then we can get $\Phi_{n}(x) \in C^{2}(\mathbb{R})$ with $\Phi_{n}^{\prime \prime}(x)=\psi_{n}(x),-1 \leq \Phi_{n}^{\prime}(x)=$ $\int_{0}^{x} \psi_{n}(z) d z \leq 0$ for $x<0$ and $\Phi_{n}(x)=0$ for $x \geq 0$. Note that, for $x<0$ there exists $n_{0} \in \mathbb{N}$ such that for all $n \geq n_{0}$ we have $a_{n}>x$. About $\Phi_{n}$ we can get the following properties as $n \rightarrow \infty$,

$$
\Phi_{n}(x) \rightarrow-\min \{x, 0\}=: \phi(x), \Phi_{n}^{\prime}(x) \rightarrow-1(x<0) .
$$


By ItO's formula

$$
\begin{aligned}
\Phi_{n}\left(X_{\varepsilon}(t, x)\right)= & \Phi_{n}(f(x))+\int_{0}^{t} \Phi_{n}^{\prime}\left(X_{\varepsilon}(s, x)\right) \Delta_{\varepsilon} X_{\varepsilon}(s, x) d s \\
& +\int_{0}^{t} \Phi_{n}^{\prime}\left(X_{\varepsilon}(s, x)\right) a\left(X_{\varepsilon}(s, x)\right) d W_{x}^{\varepsilon}(s) \\
& +\frac{1}{2} \int_{0}^{t} \Phi^{\prime \prime}\left(X_{\varepsilon}(s, x)\right) a^{2}\left(X_{\varepsilon}(s, x)\right) d s
\end{aligned}
$$

From the Lipschitz condition and $a(0)=0$,

$$
\Phi^{\prime \prime}\left(X_{\varepsilon}(s, x)\right) a\left(X_{\varepsilon}(s, x)\right)^{2} \leq C \Phi^{\prime \prime}\left(X_{\varepsilon}(s, x)\right) X_{\varepsilon}^{2}(s, x) \leq C / n .
$$

Hence

$$
E\left[\Phi_{n}\left(X_{\varepsilon}(s, x)\right)\right] \leq E \int_{0}^{t} \Phi_{n}^{\prime}\left(X_{\varepsilon}(s, x)\right) \Delta_{\varepsilon} X_{\varepsilon}(s, x) d s+\frac{C}{n} t .
$$

Taking the limit as $n \rightarrow \infty$ and by monotone convergence theorem

$$
\begin{aligned}
E\left[\phi\left(X_{\varepsilon}(t, x)\right)\right] \leq & E \int_{0}^{t}-1\left(X_{\varepsilon}(s, x)<0\right) \Delta_{\varepsilon} X_{\varepsilon}(s, x) d s \\
= & \frac{1}{\varepsilon} \int_{0}^{t} E\left[1\left(X_{\varepsilon}<0\right) X_{\varepsilon}(s, x)\right] d s \\
& -\frac{1}{\varepsilon} \int_{0}^{t} \int_{\mathbb{R}} G(\varepsilon, x, y) E\left[1\left(X_{\varepsilon}(s, x)<0\right) X_{\varepsilon}(s, y)\right] d y d s
\end{aligned}
$$

For the second term, we notice that

$$
\begin{aligned}
- & \frac{1}{\varepsilon} \int_{0}^{t} \int_{\mathbb{R}} G(\varepsilon, x, y) E\left[1\left(X_{\varepsilon}(s, x)<0\right) X_{\varepsilon}(s, y)\right] d y d s \\
& \leq-\frac{1}{\varepsilon} \int_{0}^{t} \int_{\mathbb{R}} G(\varepsilon, x, y) E\left[1\left(X_{\varepsilon}(s, x)<0, X_{\varepsilon}(s, y)<0\right) X_{\varepsilon}(s, y)\right] d y d s \\
& =\frac{1}{\varepsilon} \int_{0}^{t} \int_{\mathbb{R}} G(\varepsilon, x, y) E\left[1\left(X_{\varepsilon}(s, x)<0, X_{\varepsilon}(s, y)<0\right)\left|X_{\varepsilon}(s, y)\right|\right] d y d s \\
& \leq \frac{1}{\varepsilon} \int_{0}^{t} \int_{\mathbb{R}} G(\varepsilon, x, y) E\left[1\left(X_{\varepsilon}(s, y)<0\right)\left|X_{\varepsilon}(s, y)\right|\right] d y d s .
\end{aligned}
$$

Then, since $|x| 1(x<0)=\phi(x)$, we get

$$
E\left[\phi\left(X_{\varepsilon}(t, x)\right)\right] \leq \frac{1}{\varepsilon} \int_{0}^{t} \int_{\mathbb{R}} G(\varepsilon, x, y) E\left[\phi\left(X_{\varepsilon}(s, y)\right)\right] d y d s .
$$


Therefore, by Grownwall's lemma for $\sup _{x \in \mathbb{R}} E\left(\phi\left(X_{\varepsilon}(t, x)\right)\right)$, we obtain

$$
E\left[\phi\left(X_{\varepsilon}(t, x)\right)\right]=0
$$

for every $t>0$ and $x \in \mathbb{R}$, which yields (3.8). Let

$$
\begin{aligned}
G_{\varepsilon}(t) & =\exp t \Delta_{\varepsilon}=e^{-t / \varepsilon} \sum_{n=0}^{\infty} \frac{(t / \varepsilon)^{n}}{n !} G(n \varepsilon)=e^{-t / \varepsilon} I+R_{\varepsilon}(t) \\
R_{\varepsilon}(t, x, y) & =e^{-t / \varepsilon} \sum_{n=1}^{\infty} \frac{(t / \varepsilon)^{n}}{n !} G(n \varepsilon, x, y) .
\end{aligned}
$$

We will prove that

$$
\lim _{\varepsilon \rightarrow 0} \sup _{0 \leq t \leq T} \sup _{x \in \mathbb{R}} E\left|X_{\varepsilon}(t, x)-X(t, x)\right|^{2}=0
$$

To prove this fact, we need the following lemma (cf. Appendix of [1]).

\section{Lemma 3.2 (i)}

$$
\int_{\mathbb{R}}\left|R_{\varepsilon}(t, x, y)-G(t, x, y)\right| d y \leq e^{-t / \varepsilon}+C(\varepsilon / t)^{1 / 2} \forall t>0, \varepsilon>0 .
$$

(ii) For some $\alpha>0$ and $\beta>0$

$$
\int_{\mathbb{R}} R_{\varepsilon}(t, x, y)^{2} d y \leq C t^{-1 / \alpha} \quad \forall>0 .
$$

(iii)

$$
\lim _{\varepsilon \rightarrow \infty} \int_{0}^{t} \int_{\mathbb{R}}\left(R_{\varepsilon}(t, x, y)-G(t, x, y)\right)^{2}=0 \quad \forall t>0, x \in \mathbb{R} .
$$

Notice that, $X_{\varepsilon}(t, x)$ can be written in the following mild form:

$$
\begin{aligned}
& X_{\varepsilon}(t, x)=\int_{\mathbb{R}} G_{\varepsilon}(t, x, y) f(y) d y+\int_{0}^{t} \int_{\mathbb{R}} e^{-\frac{(t-s)}{\varepsilon}} a\left(u_{\varepsilon}(s, x)\right) d W_{x}^{\varepsilon}(s) \\
& \quad+\int_{0}^{t} \int_{\mathbb{R}} R_{\varepsilon}(t-s, x, y) a\left(X_{\varepsilon}(s, y)\right) d W_{y}^{\varepsilon}(s)
\end{aligned}
$$

where the last term equals to

$$
\int_{0}^{t} \int_{\mathbb{R}}\left(\int_{\mathbb{R}} R_{\varepsilon}(t-s, x, z) a\left(X_{\varepsilon}(s, z)\right) \rho_{\varepsilon}(y-z) d z\right) W(d y, d s) .
$$


Since $f$ is bounded, it follows that for every $T>0$ (see Remark 3.1),

$$
\begin{aligned}
& \sup _{0<\varepsilon \leq 1} \sup _{0 \leq t \leq T} \sup _{x \in \mathbb{R}} E\left[\left|X_{\varepsilon}(t, x)\right|^{2}\right]<\infty \\
& \sup _{0 \leq t \leq T} \sup _{x \in \mathbb{R}} E\left[|X(t, x)|^{2}\right]<\infty .
\end{aligned}
$$

Then we have

$$
\begin{aligned}
E & {\left[\left|X_{\varepsilon}(t, x)-X(t, x)\right|^{2}\right] \leq C\left\{G_{\varepsilon}(t) f(x)-\left.G(t) f(x)\right|^{2}\right.} \\
& +E\left[\int_{0}^{t} e^{-2(t-s) / \varepsilon}\left|a\left(X_{\varepsilon}(s, x)\right)\right|^{2} d s\right] \\
& +\int_{0}^{t} \int_{\mathbb{R}} E\left[\left|\int_{\mathbb{R}} R_{\varepsilon}(t-s, x, y)\left[a\left(X X_{\varepsilon}(s, z)\right)-a(X(s, z))\right] \rho_{\varepsilon}(y-z) d z\right|^{2} d y d s\right] \\
& +\int_{0}^{t} \int_{\mathbb{R}} E\left[\left|\int_{\mathbb{R}} R_{\varepsilon}(t-s, x, y)[a(X(s, z))-a(X(s, y))] \rho_{\varepsilon}(y-z) d z\right|^{2} d y d s\right] \\
& +\int_{0}^{t} \int_{\mathbb{R}} E\left[\left|\int_{\mathbb{R}}\left[R_{\varepsilon}(t-s, x, z)-G(t-s, x, z)\right] a(X(s, y)) \rho_{\varepsilon}(y-z) d z\right|^{2} d y d s\right] \\
& \left.+\int_{0}^{t} \int_{\mathbb{R}} E\left[\left|\int_{\mathbb{R}}[G(t-s, x, z)-G(t-s, x, y)] a(X(s, y)) \rho_{\varepsilon}(y-z) d z\right|^{2} d y d s\right]\right\} \\
= & C \sum_{i=1}^{6} I_{n}(t, x, \varepsilon) .
\end{aligned}
$$

By using Lemma 3.2 and boundness of $f(x)$

$$
\begin{aligned}
I_{1}(t, x, \varepsilon) & =C\left|\int_{\mathbb{R}} R_{\varepsilon}(t, x, y) f(y) d y-\int_{\mathbb{R}} G(t, x, y) f(y) d y\right|^{2} \\
& \leq C\left(\int_{\mathbb{R}}\left|R_{\varepsilon}(t, x, y)-G(t, x, y)\right| d y\right)^{2} \\
& \leq C\left(e^{\frac{-2 t}{\varepsilon}}+(\varepsilon / t)^{\frac{2}{3}}\right) .
\end{aligned}
$$

As for $I_{2}(t, x, \varepsilon)$

$$
I_{2}(t, x, \varepsilon) \leq C \int_{0}^{t} e^{-2(t-s) / \varepsilon} E\left[X_{\varepsilon}(s, x)^{2}\right] d s .
$$

This inequality and (3.9) imply that

$$
\lim _{\varepsilon \rightarrow 0} \sup _{0 \leq t \leq T} \sup _{x \in \mathbb{R}} I_{2}(t, x, \varepsilon)=0 .
$$


By the Hölder's inequality, the Lipschitz continuty of function $a(x)$ and Lemm 3.2, we have

$$
\begin{aligned}
I_{3}(t, x, \varepsilon) & \leq C \int_{0}^{t} \int_{\mathbb{R}} E\left(\int_{\mathbb{R}} R_{\varepsilon}(t-s, x, z)\left|X_{\varepsilon}(s, z)-X(s, z)\right| \rho_{\varepsilon}(y-z) d z\right)^{2} d y d s \\
& \leq C \int_{0}^{t} \int_{\mathbb{R}} E\left(\int_{\mathbb{R}} R_{\varepsilon}(t-s, x, z)^{2}\left|X_{\varepsilon}(s, z)-X(s, z)\right|^{2} \rho_{\varepsilon}(y-z) d z\right) d y d s \\
& \leq C \int_{0}^{t}(t-s)^{-\frac{1}{\alpha}} \sup _{y \in \mathbb{R}} E\left[\left|X_{\varepsilon}(s, y)-X(s, y)\right|^{2}\right] d s,
\end{aligned}
$$

and similarly

$$
I_{4}(t, x, \varepsilon) \leq C \int_{0}^{t} \int_{\mathbb{R}} \int_{\mathbb{R}} G(t-s, x, z)^{2} E\left[|X(s, z)-X(s, y)|^{2}\right] \rho_{\varepsilon}(y-z) d y d z d s .
$$

According to the inequality

$$
E\left[|X(s, z)-X(s, y)|^{2} \leq C\left\{s^{-1 / \alpha}|y-z|+|y-z|^{\alpha-1}\right\}\right.
$$

the definition of $\rho_{\varepsilon}$ gives that

$$
\begin{aligned}
I_{4}(t, x, \varepsilon) & \leq C \int_{0}^{t} \int_{\mathbb{R}} \int_{\mathbb{R}} G(t-s, x, z)^{2}\left[s^{-1 / \alpha}|y-z|+|y-z|^{\alpha-1}\right] \rho_{\varepsilon}(y-z) d y d z d s \\
& \leq C \int_{0}^{t} \int_{\mathbb{R}} G(t-s, x, y)^{2}\left[s^{-1 / \alpha} \varepsilon+\varepsilon^{\alpha-1}\right] \\
& \leq C \int_{0}^{t}(t-s)^{-1 / \alpha}\left[s^{-1 / \alpha} \varepsilon+\varepsilon^{\alpha-1}\right] \leq C\left(t^{1-2 / \alpha} \varepsilon+\varepsilon^{\alpha-1}\right) .
\end{aligned}
$$

By Hölder's inequality and (3.10),

$$
I_{5}(t, x, \varepsilon) \leq C \int_{0}^{t} \int_{\mathbb{R}} \int_{\mathbb{R}}\left[\left|R_{\varepsilon}(t-s, x, z)-G(t-s, x, z)\right|^{2} \rho_{\varepsilon}(y-z) d z\right] d y d s,
$$

and applying Lemma 3.2 we obtain

$$
\lim _{\varepsilon \rightarrow 0} \sup _{0 \leq t \leq T} \sup _{x \in \mathbb{R}} I_{5}(t, x, \varepsilon)=0 .
$$


In a same way, we have

$$
\begin{aligned}
I_{6}(t, x, \varepsilon) \leq & C \int_{0}^{t} \int_{\mathbb{R}} \int_{\mathbb{R}}\left[|G(t-s, x, z)-G(t-s, x, y)|^{2} \rho_{\varepsilon}(y-z) d z\right] d y d s \\
= & C \int_{0}^{t} \int_{\mathbb{R}}\left[G(t-s, x, y)^{2}-\int_{\mathbb{R}}\left\{2 G(t-s, x, y) G(t-s, x, z) \rho_{\varepsilon}(y-z)\right.\right. \\
& \left.\left.+G(t-s, x, z)^{2} \rho_{\varepsilon}(y-z)\right\} d z\right] d y d s .
\end{aligned}
$$

Applying the fact

$$
\lim _{\varepsilon \rightarrow 0} \int_{\mathbb{R}} G(t-s, x, z) \rho_{\varepsilon}(y-z) d z=G(t-s, x, y) \text { uniformly for } y \in \mathbb{R},
$$

we can get

$$
\lim _{\varepsilon \rightarrow 0} \sup _{0 \leq t \leq T} \sup _{x \in \mathbb{R}} I_{6}(t, x, \varepsilon)=0 .
$$

Now, we set

$$
M(t, \varepsilon)=\sup _{x \in \mathbb{R}}\left\|X_{\varepsilon}(t, x)-X(t, x)\right\|_{2}^{2} .
$$

Then there exists some constant $C>0$ such that

$$
M(t, \varepsilon) \leq C \int_{0}^{t}(t-s)^{-1 / \alpha} M(s, \varepsilon)+H(T, \varepsilon)+\hat{H}(t, \varepsilon),
$$

where

$$
\begin{aligned}
& H(T, \varepsilon)=C \sum_{n=2,5,6} \sup _{0 \leq t \leq T} \sup _{x \in \mathbb{R}} I_{n}(t, x, \varepsilon), \\
& \hat{H}(t, x, \varepsilon)=C\left\{\left(e^{-t / \varepsilon}+(\varepsilon / t)^{1 / 2}\right)+\left(t^{1-2 / \alpha}+\varepsilon^{\alpha-1}\right)\right\} .
\end{aligned}
$$

Therefore, Grownwall's inequality implies that

$$
M(t, \varepsilon) \rightarrow 0 \text { as } \varepsilon \rightarrow 0
$$

and thus completes the proof of Theorem 3.3.

\subsection{Polynomial decay}

In this section, we show that the solution of (3.1) has modification in the class $C_{\rho}(\mathbb{R})$. The following lemma is a variant of a Kolmogorov's continuity criterion theorem. 
Lemma 3.3 (i) Suppose that for every $0<\rho<(\alpha+1) / 2$ there exist $p>0, \gamma>2$ and $C_{\rho}>0$ such that

$$
E\left[\left|X(t, x)-X\left(t^{\prime}, x^{\prime}\right)\right|^{p}\right] \leq C_{\rho}\left(\left|t-t^{\prime}\right|^{\gamma}+\left|x-x^{\prime}\right|^{\gamma}\right) \lambda^{-\rho}(x),
$$

for $0 \leq t, t^{\prime} \leq 1$ and $x, x^{\prime} \in \mathbb{R}$ with $\left|x-x^{\prime}\right| \leq 1$. Then $X(t, \cdot)$ has $C_{\rho}$-valued continuous version $P$-a.s.

(ii) Let $\left\{X_{n}(t, \cdot) ; t \geq 0, n \in \mathbb{N}\right\}$ be a sequence of continuous $C_{\rho}$-valued processes. Suppose that for every $0<\rho<(\alpha+1) / 2$ and $T>0$ there exists $p>0, \gamma>2$ and $C_{\rho}>0$ such that

$$
E\left[\left|X_{n}(t, x)-X_{n}\left(t^{\prime}, x^{\prime}\right)\right|^{p}\right] \leq C_{\rho}\left(\left|t-t^{\prime}\right|^{\gamma}+\left|x-x^{\prime}\right|^{\gamma}\right) \lambda^{-\rho}(x),
$$

for $t, t^{\prime} \in[0, T]$ and $x, x^{\prime} \in \mathbb{R}$ with $\left|x-x^{\prime}\right| \leq 1$. Then the sequence of probability distributions on $C\left([0, \infty) ; C_{\rho}(\mathbb{R})\right)$ induced by $X_{n}(\cdot)$ is tight.

Theorem 3.4 Under the conditions of Lemma 3.1, X( $t, \cdot)$ has $C_{\rho}(\mathbb{R})$ valued continuous version $P$-a.s.

Proof Since $f \in C_{\rho}(\mathbb{R})$, it is enough to prove that for all $0<\rho<(\alpha+1) / 2$ there exist $p>0, \gamma>2$ and $C_{\rho}>0$ such that

$$
E\left[\left|X(t, x)-X\left(t^{\prime}, x^{\prime}\right)\right|^{p}\right] \leq C\left(\left|t-t^{\prime}\right|^{\gamma}+\left|x-x^{\prime}\right|^{\gamma}\right) \lambda^{-\rho}(x),
$$

for $X(t, x)=\int_{0}^{t} \int_{\mathbb{R}} G(t-s, x, y) a(X(s, y)) W(d y, d s), 0 \leq t, t^{\prime} \leq 1$ and $x, x^{\prime} \in \mathbb{R}$ with $\left|x-x^{\prime}\right| \leq 1$. We first show (3.11) with $t=t^{\prime}$. From the Burkholder's inequality and the Hölder's inequality, we have for every $p=2^{m}, m \in \mathbb{N}$,

$$
\begin{aligned}
E & {\left[\left|X(t, x)-X\left(t, x^{\prime}\right)\right|^{p}\right] } \\
\leq & C_{p} E\left[\int_{0}^{t} \int_{\mathbb{R}}\left(G(t-s, x, y)-G\left(t-s, x^{\prime}, y\right)\right)^{2} a(X(s, y))^{2} d y d s\right]^{\frac{p}{2}} \\
\leq & C_{p} E\left(\int_{0}^{t} \int_{\mathbb{R}}\left|G(t-s, x, y)-G\left(t-s, x^{\prime}, y\right)\right|^{2} a(X(s, y))^{p} \lambda^{\rho\left(\frac{p}{2}-1\right)}(y) d y d s\right) \\
& \times\left(\int_{0}^{t} \int_{\mathbb{R}}\left|G(t-s, x, y)-G\left(t-s, x^{\prime}, y\right)\right|^{2} \lambda^{-\rho}(y) d y d s\right)^{\frac{p-2}{2}},
\end{aligned}
$$

where $\rho<\frac{\alpha+1}{p-2}$. Lemma 3.1 implies that

$E\left(\int_{0}^{t} \int_{\mathbb{R}}\left|G(t-s, x, y)-G\left(t-s, x^{\prime}, y\right)\right|^{2} a(X(s, y))^{p} \lambda^{\rho\left(\frac{p}{2}-1\right)}(y) d y d s\right)<\infty$. 
From Lemma 2.1

$$
\begin{aligned}
\left|G(t, x, y)-G\left(t, x^{\prime}, y\right)\right| & \leq C\left|x-x^{\prime}\right| G_{x}(t, x(\theta), y) \\
& =C\left|x-x^{\prime}\right| t^{\frac{-2}{\alpha}} G_{x}\left(1,(x(\theta)-y) t^{\frac{-1}{\alpha}}\right) \\
& \leq C\left|x-x^{\prime}\right| t^{\frac{-2}{\alpha}}
\end{aligned}
$$

where $x(\theta)=x+(1-\theta) x^{\prime}$ for $0<\theta<1$. Therefore, for every $\kappa<\alpha-1$

$$
\begin{aligned}
\int_{0}^{t} & \int_{\mathbb{R}}\left|G(t-s, x, y)-G\left(t-s, x^{\prime}, y\right)\right|^{2-\kappa+\kappa} \lambda^{-\rho}(y) d y d s \\
\leq & C\left|x-x^{\prime}\right|^{\kappa} \int_{0}^{t}(t-s)^{-\frac{2}{\alpha} \kappa} \\
& \int_{\mathbb{R}}\left(|G(t-s, x, y)|^{2-\kappa}+\left|G\left(t-s, x^{\prime}, y\right)\right|^{2-\kappa}\right) \lambda^{-\rho}(y) d y d s .
\end{aligned}
$$

Note that,

$$
\int_{\mathbb{R}} G(t, x, y)^{2-\kappa} \lambda^{-\rho}(y) d y \leq C t^{-\frac{1-\kappa}{\alpha}} \lambda^{-\rho}(x),
$$

so we have

$$
\begin{aligned}
& \int_{0}^{t} \int_{\mathbb{R}}\left|G(t-s, x, y)-G\left(t-s, x^{\prime}, y\right)\right|^{2-\kappa+\kappa} \lambda^{-\rho}(y) d y d s \\
& \quad \leq C\left|x-x^{\prime}\right|^{\kappa} \int_{0}^{t}(t-s)^{-\frac{1+\kappa}{\alpha}} d s \lambda^{-\rho}(x) .
\end{aligned}
$$

Choosing $p=2^{m}$ satisfying that $\frac{p-2}{2} \kappa>2$, we can get (3.11) with $t=t^{\prime}$. Next, we prove (3.11) with $x=x^{\prime}$. In the same way as above, for $0 \leq t \leq t^{\prime} \leq T$, we can show that

$$
\begin{aligned}
E & {\left[\left|X(t, x)-X\left(t^{\prime}, x\right)\right|^{p}\right] } \\
\leq & C_{p}\left\{E\left[\int_{0}^{t} \int_{\mathbb{R}}\left(G\left(t^{\prime}-s, x, y\right)-G(t-s, x, y)\right)^{2} a(X(s, y))^{2} d y d s\right]^{\frac{p}{2}}\right. \\
& \left.+E\left[\int_{t}^{t^{\prime}} \int_{\mathbb{R}} G\left(t^{\prime}-s, x, y\right)^{2} a(X(s, y))^{2} d y d s\right]^{\frac{p}{2}}\right\} \\
\leq & C_{p}\left\{E\left(\int_{t}^{t^{\prime}} \int_{\mathbb{R}} G\left(t^{\prime}-s, x, y\right)^{2} a(X(s, y))^{p} \lambda^{\rho\left(\frac{p}{2}-1\right)}(y) d y d s\right)\right.
\end{aligned}
$$




$$
\begin{aligned}
& \times\left(\int_{t}^{t^{\prime}} \int_{\mathbb{R}}\left|G\left(t^{\prime}-s, x, y\right)\right|^{2} \lambda^{-\rho}(y) d y d s\right)^{\frac{p-2}{2}} \\
& +E\left(\int_{0}^{t} \int_{\mathbb{R}}\left|G\left(t^{\prime}-s, x, y\right)-G(t-s, x, y)\right|^{2} a(X(s, y))^{p} \lambda^{\rho\left(\frac{p}{2}-1\right)}(y) d y d s\right) \\
& \left.\times\left(\int_{0}^{t} \int_{\mathbb{R}}\left|G\left(t^{\prime}-s, x, y\right)-G(t-s, x, y)\right|^{2} \lambda^{-\rho}(y) d y d s\right)^{\frac{p-2}{2}}\right\},
\end{aligned}
$$

for $\rho<\frac{\alpha+1}{p-2}$. Immediately, we can get from Lemma 2.1

$$
\begin{aligned}
\int_{t}^{t^{\prime}} \int_{\mathbb{R}}\left|G\left(t^{\prime}-s, x, y\right)\right|^{2} \lambda^{-\rho}(y) d y d s & \leq C \int_{t}^{t^{\prime}}\left(t^{\prime}-s\right)^{-1 / \alpha} \int_{\mathbb{R}} G(t-s, x, y) \lambda^{-\rho}(y) d y \\
& \leq C\left(t-t^{\prime}\right)^{1-\frac{1}{\alpha}} \lambda^{-\rho}(x) .
\end{aligned}
$$

Hence combining with (3.5)

$$
\begin{aligned}
& C_{p} E\left(\int_{t}^{t^{\prime}} \int_{\mathbb{R}} G\left(t^{\prime}-s, x, y\right)^{2} a(X(s, y))^{p} \lambda^{-\rho}(y) d y d s\right) \\
& \quad \times\left(\int_{t}^{t^{\prime}} \int_{\mathbb{R}}\left|G\left(t^{\prime}-s, x, y\right)\right|^{2} \lambda^{-\rho}(y) d y d s\right)^{\frac{p-2}{2}} \\
& \leq C_{p, T, \alpha}\left(t-t^{\prime}\right)^{\left(1-\frac{1}{\alpha}\right) \frac{p-2}{2}} \lambda^{-\rho \frac{p-2}{2}}(x) .
\end{aligned}
$$

From now on, we claim that

$$
\int_{0}^{t} \int_{\mathbb{R}}\left|G\left(t^{\prime}-s, x, y\right)-G(t-s, x, y)\right|^{2} \lambda^{-\rho}(y) d y d s \leq\left(t-t^{\prime}\right)^{\left(1-\frac{1}{\alpha}\right)} \lambda^{-\rho}(x) .
$$

The change of variable $s=\theta v$ with $\theta=t^{\prime}-t$,

$$
\begin{aligned}
& \int_{0}^{t} \int_{\mathbb{R}}\left|G\left(t^{\prime}-t+s, x, y\right)-G(s, x, y)\right|^{2} \lambda^{-\rho}(y) d y d s \\
& =\int_{0}^{t / \theta} \int_{\mathbb{R}}|G(\theta(v+1), x, y)-G(\theta v, x, y)|^{2} \lambda^{-\rho}(y) \theta d y d v \\
& \leq \int_{0}^{t / \theta} \int_{\mathbb{R}} \theta^{1-\frac{2}{\alpha}} \mid G\left(v+1, \theta^{-\frac{1}{\alpha}}(x-y)\right) \\
& \quad-\left.G\left(v, \theta^{-\frac{1}{\alpha}}(x-y)\right)\right|^{2} \lambda^{-\rho}(y) d y d v .
\end{aligned}
$$

Note that,

$$
\lambda(y)=\left(1+|y|^{2}\right)^{1 / 2}=\left(1+\left|\theta^{\frac{1}{\alpha}} \theta^{-\frac{1}{\alpha}} y\right|^{2}\right)^{1 / 2} \leq C\left(1+\left|\theta^{-\frac{1}{\alpha}} y\right|^{2}\right)^{1 / 2} .
$$


Again, by the change of variable $z=\theta^{-\frac{1}{\alpha}}(x-y)$, we have

$$
\begin{aligned}
& \int_{0}^{t} \int_{\mathbb{R}}\left|G\left(t^{\prime}-s, x, y\right)-G(t-s, x, y)\right|^{2} \lambda^{-\rho}(y) d y d v \\
& \quad \leq \theta^{1-\frac{1}{\alpha}} \lambda^{-\rho}(x) \int_{0}^{t / \theta} \int_{\mathbb{R}}|G(v+1, z)-G(v, z)|^{2} \lambda^{\rho}(z) d z d v .
\end{aligned}
$$

Therefore, to prove (3.11) we need to show that

$$
\int_{0}^{\infty} \int_{\mathbb{R}}|G(v+1, z)-G(v, z)|^{2} \lambda^{\rho}(z) d z d v<\infty .
$$

Let us write

$$
\begin{aligned}
\int_{0}^{\infty} & \int_{\mathbb{R}}|G(v+1, z)-G(v, z)|^{2} \lambda^{\rho}(z) d z d v \\
= & \int_{0}^{1} \int_{\mathbb{R}}|G(v+1, z)-G(v, z)|^{2} \lambda^{\rho}(z) d z d v \\
& +\int_{1}^{\infty} \int_{\mathbb{R}}|G(v+1, z)-G(v, z)|^{2} \lambda^{\rho}(z) d z d v
\end{aligned}
$$

The first integral is finite, since

$$
\begin{aligned}
& \int_{0}^{1} \int_{\mathbb{R}}|G(v+1, z)-G(v, z)|^{2} \lambda^{\rho}(z) d z d v \\
& \quad \leq C \int_{0}^{1} \int_{\mathbb{R}} v^{-\frac{1}{\alpha}}|G(v, z)| \lambda^{\rho}(z) d z d v<\infty .
\end{aligned}
$$

For the second term one, we use Lemma 2.1 and the change of variable $z=(1+v)^{1 / \alpha} z^{\prime}$

$$
\begin{aligned}
& \int_{1}^{\infty} \int_{\mathbb{R}}|G(v+1, z)-G(v, z)|^{2} \lambda^{\rho}(z) d z d v \\
& =\int_{1}^{\infty} \int_{\mathbb{R}}\left|G\left(1,(1+v)^{-\frac{1}{\alpha}} z\right) \times(1+v)^{-\frac{1}{\alpha}}-G\left(1, v^{-\frac{1}{\alpha}} z\right) \times v^{-\frac{1}{\alpha}}\right|^{2} \lambda^{\rho}(z) d z d v \\
& \quad=\int_{1}^{\infty} \int_{\mathbb{R}} v^{-\frac{2}{\alpha}}\left|G\left(1, z^{\prime}\right) \times\left(\frac{v}{1+v}\right)^{\frac{1}{\alpha}}-G\left(1,\left(\frac{1+v}{v}\right)^{\frac{1}{\alpha}} z^{\prime}\right)\right|^{2}(1+v)^{\frac{1}{\alpha}} \lambda^{\rho}\left(z^{\prime}\right) d z^{\prime} d v .
\end{aligned}
$$

Further,

$$
\begin{aligned}
& \left|G\left(1, z^{\prime}\right) \times\left(\frac{v}{1+v}\right)^{\frac{1}{\alpha}}-G\left(1,\left(\frac{1+v}{v}\right)^{\frac{1}{\alpha}} z^{\prime}\right)\right|^{2} \\
& \quad \leq C\left[\left|G\left(1, z^{\prime}\right)-G\left(1,\left(\frac{1+v}{v}\right)^{\frac{1}{\alpha}} z^{\prime}\right)\right|^{2}+\left|1-\left(\frac{v}{1+v}\right)^{\frac{1}{\alpha}}\right|^{2}\left|G\left(1, z^{\prime}\right)\right|^{2}\right],
\end{aligned}
$$


and by Lemma 2.1

$$
\begin{aligned}
& \int_{-\infty}^{\infty}\left|G(1, z)-G\left(1,\left(\frac{1+v}{v}\right)^{\frac{1}{\alpha}} z\right)\right|^{2} \lambda^{\rho}(z) d z \\
& =\int_{-\infty}^{\infty}\left|\int_{\left(\frac{1+v}{v}\right)^{\frac{1}{\alpha}} z}^{z} G_{\xi}(1, \xi) d \xi\right|^{2} \lambda^{\rho}(z) d z \\
& \leq C\left[\left.\int_{-\infty}^{-1}\left|\int_{\left.\left(\frac{1+v}{v}\right)^{\frac{1}{\alpha}} z \frac{1+|\xi|^{\alpha}}{\left(1+|\xi|^{\alpha+1}\right)^{2}} d \xi\right|^{2} \lambda^{\rho}(z) d z}^{z}\right|^{2}\right|^{\rho} \lambda^{\rho}(z) d z\right. \\
& +\int_{0}^{1}\left|\int_{z}^{\left.\left(\frac{1+v}{v}\right)^{\frac{1}{\alpha}} z\right)^{\frac{1}{\alpha}} z} \frac{1+|\xi|^{\alpha}}{\left(1+|\xi|^{\alpha+1}\right)^{2}} d \xi\right|^{2} \lambda^{\rho}(z) d z \\
& \left.+\int_{1}^{\infty}\left|\int_{z}^{\left(\frac{1+v}{v}\right)^{\frac{1}{\alpha}} z} \frac{\left.1+|\xi|^{\alpha+1}\right)^{2}}{\left(1+|\xi|^{\alpha+1}\right)^{2}} d \xi\right|^{2} \lambda^{\rho}(z) d z\right] .
\end{aligned}
$$

Notice that,

$$
\begin{gathered}
\int_{\left(\frac{1+v}{v}\right)^{\frac{1}{\alpha}} z}^{z} \frac{1+|\xi|^{\alpha}}{\left(1+|\xi|^{\alpha+1}\right)^{2}} d \xi \leq \frac{C}{\left(1+|z|^{\alpha+1}\right)^{2}} \int_{\left(\frac{1+v}{v}\right)^{\frac{1}{\alpha}} z}^{z}|\xi|^{\alpha} d \xi \quad \text { on } z \in(-\infty,-1) \\
\int_{z}^{\left(\frac{1+v}{v}\right)^{\frac{1}{\alpha}} z} \frac{1+|\xi|^{\alpha}}{\left(1+|\xi|^{\alpha+1}\right)^{2}} d \xi \leq \frac{C}{\left(1+|z|^{\alpha+1}\right)^{2}} \int_{z}^{\left(\frac{1+v}{v}\right)^{\frac{1}{\alpha}} z}|\xi|^{\alpha} d \xi \quad \text { on } z \in(1, \infty) .
\end{gathered}
$$

Thus we can estimate

$$
\begin{aligned}
& \int_{-\infty}^{\infty}\left|G(1, z)-G\left(1,\left(\frac{1+v}{v}\right)^{\frac{1}{\alpha}} z\right)\right|^{2} \lambda^{\rho}(z) d z \\
& \leq C\left(1+\int_{1}^{\infty}\left|\frac{z^{\alpha+1}}{\left(1+z^{\alpha+1}\right)^{2}}\right|^{2} \lambda^{\rho}(z) d z\right)\left|1-\left(\frac{v+1}{v}\right)^{\frac{\alpha+1}{\alpha}}\right|^{2}
\end{aligned}
$$


Since $0<\rho<(\alpha+1) / 2$, we have

$$
\begin{aligned}
& \int_{\mathbb{R}}\left|G(1, z) \times\left(\frac{v}{1+v}\right)^{\frac{1}{\alpha}}-G\left(1,\left(\frac{1+v}{v}\right)^{\frac{1}{\alpha}} z\right)\right|^{2} \lambda^{\rho}(z) d z \\
& \leq C\left(\left|1-\left(\frac{v+1}{v}\right)^{\frac{\alpha+1}{\alpha}}\right|^{2}+\left|1-\left(\frac{v+1}{v}\right)^{\frac{1}{\alpha}}\right|^{2}\right) .
\end{aligned}
$$

By the mean value theorem, we can get

$$
\left|1-\left(\frac{v+1}{v}\right)^{\frac{\alpha+1}{\alpha}}\right|^{2}+\left|1-\left(\frac{v+1}{v}\right)^{\frac{1}{\alpha}}\right|^{2} \leq C v^{-2} .
$$

Hence

$$
\begin{aligned}
& \int_{1}^{\infty} \int_{\mathbb{R}}|G(v+1, z)-G(v, z)|^{2} \lambda^{\rho}(z) d z d v \\
& \leq C \int_{1}^{\infty} v^{\frac{-2(\alpha+1)}{\alpha}}(1+v)^{1 / \alpha} d v
\end{aligned}
$$

Since $-2(\alpha+1) / \alpha+1 / \alpha<-1$, the last integral is finite and thus completes the proof of Theorem 3.4 .

\section{Existence in non-Lipschitz case}

In this section, we consider SPDE (1.1). We prove the existence of solutions by using the results in the previous section.

Theorem 4.1 Let $f \in C_{\rho}^{+}(\mathbb{R})$ be an initial function. Then for every $T>0$, there exist an $\left\{\mathcal{F}_{t}\right\}$-space-time Gaussian white noise $\dot{W}(t, x)$ and $C\left([0, T] ; C_{\rho}^{+}(\mathbb{R})\right)$ valued solutions $X$ to $(2.3)$ with $X(0)=f$ on a suitable probability space with filtration $\left(\Omega, \mathcal{F}, P,\left\{\mathcal{F}_{t}\right\}\right)$.

Proof Let $a_{n}(u)$ be a sequence of Lipschitz functions such that

$$
a_{n}(u)=\left\{\begin{aligned}
n^{1-\gamma}|u| & \text { if }|u|<\frac{1}{n}, \\
|u|^{\gamma} & \text { if }|u| \geq \frac{1}{n}
\end{aligned}\right.
$$

The sequence $a_{n}(u)$ converge to $|u|^{\gamma}$ uniformly in $u \in \mathbb{R}$ as $n \rightarrow \infty$. Then by Theorem 3.1, 3.3 and 3.4 for every $0<\rho<(\alpha+1) / 2$, there exist the unique $C_{\rho}^{+}(\mathbb{R})$ valued solution $X_{n}$ to (3.1) with $a_{n}(u)$ for each $n \geq 1$.

The solution $X_{n}$ holds the moment condition (3.11), and it follows from Lemma 3.3 that the family of probability distributions on $C\left([0, T] ; C_{\rho}^{+}(\mathbb{R})\right)$ induced by $\left\{X_{n}\right\}$ is 
tight. This means that there exists a subsequence $\left\{n_{k}\right\}_{k \in \mathbb{N}}$ and a random field $X \in$ $C\left([0, T] ; C_{\rho}^{+}(\mathbb{R})\right)$ such that

$$
X_{n_{k}} \Rightarrow X \text { on } C\left([0, T] ; C_{\rho}^{+}(\mathbb{R})\right)
$$

By Skorohod representation theorem (cf. [5]), we can find some random fields $Y_{n}, Y \in$ $C\left([0, T] ; C_{\rho}^{+}(\mathbb{R})\right)$ on some probability space $\left(\bar{\Omega}, \overline{\mathcal{F}},\left(\overline{\mathcal{F}}_{t}\right)_{0 \leq t \leq T}, \bar{P}\right)$, such that

$$
Y_{n} \rightarrow Y \quad \bar{P}-\text { a.s. on } C\left([0, T] ; C_{\rho}^{+}(\mathbb{R})\right)
$$

and

$$
X_{n}=Y_{n} \text { in law, } \quad X=Y \text { in law. }
$$

Then we can get for every $\phi \in \mathcal{D}\left((-\Delta)^{\alpha / 2}\right)$

$$
\begin{aligned}
M_{\phi}^{n}(t):= & \int_{0}^{t} \int_{\mathbb{R}} \phi(y) a_{n}\left(X_{n}(s, y)\right) W(d y, d s) \\
= & \int_{\mathbb{R}} X_{n}(t, y) \phi(y) d y+\int_{0}^{t} \int_{\mathbb{R}}(-\Delta)^{\alpha / 2} \phi(y) X_{n}(s, y) d y d s \\
& \quad-\int_{\mathbb{R}} \phi(y) f(y) d y \\
& \stackrel{\text { in law }}{=} \int_{\mathbb{R}} Y_{n}(t, y) \phi(y) d y+\int_{0}^{t} \int_{\mathbb{R}}(-\Delta)^{\alpha / 2} \phi(y) Y_{n}(s, y) d y d s \\
& -\int_{\mathbb{R}} \phi(y) f(y) d y \\
\rightarrow & \int_{\mathbb{R}} X(t, y) \phi(y) d y+\int_{0}^{t} \int_{\mathbb{R}}(-\Delta)^{\alpha / 2} \phi(x) X(s, y) d y d s \\
& -\int_{\mathbb{R}} \phi(y) f(y) d y
\end{aligned}
$$

Note that, by (3.7)

$$
\sup _{0 \leq t \leq T} \sup _{n \in \mathbb{N}} E\left[\left|M_{\phi}^{n}(t)\right|^{2}\right]<\infty
$$

Hence, $\left(M_{\phi}^{n}\right)_{n \in \mathbb{N}}$ is a sequence of uniformly integrable martingales, and therefore, there exists a martingale $M_{\phi}$ such that

$$
M_{\phi}^{n}(\cdot) \Rightarrow M_{\phi}(\cdot)
$$

and

$M_{\phi}(t)=\int_{\mathbb{R}} X(t, y) \phi(y) d y+\int_{0}^{t} \int_{\mathbb{R}}(-\Delta)^{\alpha / 2} \phi(y) X(s, y) d y d s-\int_{\mathbb{R}} \phi(y) f(y) d y$. 
It follows from (4.1) we can get as $n \rightarrow \infty$,

$$
\left\langle M_{\phi}^{n}, M_{\phi}^{n}\right\rangle_{t} \rightarrow \int_{0}^{t} \int_{\mathbb{R}} \phi^{2}(y)|X(s, y)|^{2 \gamma} d y d s,
$$

and so that

$$
\left\langle M_{\phi}, M_{\phi}\right\rangle_{t}=\int_{0}^{t} \int_{\mathbb{R}} \phi^{2}(y)|X(s, y)|^{2 \gamma} d y d s .
$$

This means that there corresponds a martingale measure $M(d x, d t)$ with the quadratic measure

$$
Q(d t, d y)=|X(t, y)|^{2 \gamma} d y d t
$$

Take an $\mathcal{S}^{\prime}(\mathbb{R})$-valued standard Wiener process $\bar{W}_{t}$ independent of $X_{S}(d x)$. We set

$$
\begin{aligned}
W_{t}(\phi)= & \int_{0}^{t} \int_{\mathbb{R}} \frac{1}{|X(s, y)|^{\gamma}} 1_{\{X(s, y) \neq 0\}} \phi(y) M(d y, d s) \\
& +\int_{0}^{t} \int_{\mathbb{R}} 1_{\{X(s, y)=0\}} \phi(y) \bar{W}(d y, d s) .
\end{aligned}
$$

From the definition of $M$ and $W$ we can show that

$$
\begin{gathered}
\int_{0}^{t} \int_{\mathbb{R}} \phi(y)|X(s, y)|^{\gamma} W(d y, d s)=\int_{0}^{t} \int_{\mathbb{R}} \phi(y) 1_{\{X(s, y) \neq 0\}} M(d y, d s) \\
=\int_{0}^{t} \int_{\mathbb{R}} \phi(y) M(d y, d s)-\int_{0}^{t} \int_{\mathbb{R}} \phi(y) 1_{\{X(s, y)=0\}} M(d y, d s) .
\end{gathered}
$$

Since the last term equals to 0 a.s., we have

$$
M_{\phi}(t)=\int_{0}^{t} \int_{\mathbb{R}}|X(s, y)|^{\gamma} \phi(y) W(d y, d s) .
$$

Thus we complete the proof of Theorem 4.1.

Open Access This article is licensed under a Creative Commons Attribution 4.0 International License, which permits use, sharing, adaptation, distribution and reproduction in any medium or format, as long as you give appropriate credit to the original author(s) and the source, provide a link to the Creative Commons licence, and indicate if changes were made. The images or other third party material in this article are included in the article's Creative Commons licence, unless indicated otherwise in a credit line to the material. If material is not included in the article's Creative Commons licence and your intended use is not permitted by statutory regulation or exceeds the permitted use, you will need to obtain permission directly from the copyright holder. To view a copy of this licence, visit http://creativecommons.org/licenses/by/4.0/. 


\section{References}

1. Chen, L., Kim, K.: On comparison principle and strict positivity of solutions to the nonlinear stochastic fractional heat equations. Ann. Inst. Henri Poincare Probab. Stat. 53(1), 358-388 (2017). https://doi. org/10.1214/15-AIHP719

2. Da Prato, G., Zabczyk, J.: Stochastic Equations in Infinite Dimensions. Cambridge University Press, Cambridge (1992)

3. Dawson, D.: The critical measure diffusion process. Z. Wahr. Verw Gebiete 40, 125-145 (1977)

4. Debbi, L., Dozzi, M.: On the solutions of nonlinear stochastic fractional partial differential equations in one spatial dimension. Stoch. Process. Appl. 115(11), 1764-1781 (2005)

5. Ethier, S., Kurtz, T.: Markov Processes. Characterization and Convergence. Wiley Series in Probability and Mathematical Statistics. Wiley, New York (1986)

6. Fleischmann, K.: Critical behavior of some measure-valued processes. Math. Nachr. 135, 131-147 (1988)

7. Funaki, T.: Regularity properties for stochastic partial differential equations of parabolic type. Osaka J. Math. 28(3), 495-516 (1991)

8. Iscoe, I.: A weighted occupation time for a class of measure-valued branching processes. Probab. Theory Relat. Fields 71, 85-116 (1986)

9. Kotelenez, P.: Existence, uniqueness and smoothness for a class of function valued stochastic partial differential equations. Stoch. Stoch. Rep. 41, 177-199 (1992)

10. Kunita, H.: Stochastic Flows and Stochastic Differential Equations. Cambridge University Press, Cambridge (1997)

11. Mueller, C., Perkins, E.: The compact support property for solutions to the heat equation with noise. Probab. Theory Relat. Fields 93, 325-358 (1992)

12. Mueller, C.: The heat equation with Lévy noise. Stoch. Process. Appl. 74(1), 67-82 (1998)

13. Mytnik, L.: Weak uniqueness for the heat equation with noise. Ann. Probab. 26, 968-984 (1998)

14. Mytnik, L.: Stochastic partial differential equation driven by stable noise. Probab. Theory Relat. Fields 123(2), 157-201 (2002)

15. Niu, M., Xie, B.: Regularity of a fractional partial differential equation driven by space-time white noise. Proc. Am. Math. Soc. 138, 1479-1489 (2010)

16. Peszat, S., Zabczyk, J.: Stochastic Partial Differential Equations with Lévy Noise. An Evolution Equation Approach, Encyclopedia of Mathematics and its Applications, 113. Cambridge University Press, Cambridge (2007)

17. Shiga, T.: Two contrasting properties of solutions for one-dimensional stochastic partial differential equations. Can. J. Math. 46(2), 415-437 (1994)

18. Walsh, J.: An Introduction to Stochastic Partial Differential Equations. Ecole d'Eté de probabilités de Saint-Flour, XIV-1984, Lecture Notes in Mathematics, vol. 1180, pp. 265-439. Springer, Berlin (1986)

Publisher's Note Springer Nature remains neutral with regard to jurisdictional claims in published maps and institutional affiliations. 